\title{
Dynamic Analysis of a Competition-Cooperation Enterprise Cluster with Core-Satellite Structure and Time Delay
}

\author{
Wenjie Hu, ${ }^{1}$ Tao Dong $\mathbb{D}^{2}{ }^{2}$ and Hua Zhao ${ }^{1}$ \\ ${ }^{1}$ School of Business Administration, Chongqing Technology and Business University, Chongqing 400067, China \\ ${ }^{2}$ College of Electronics and Information Engineering, Southwest University, Chongqing 400715, China
}

Correspondence should be addressed to Tao Dong; david_312@126.com

Received 2 November 2020; Revised 3 April 2021; Accepted 12 May 2021; Published 2 June 2021

Academic Editor: Jiaojiao Jiang

Copyright (c) 2021 Wenjie Hu et al. This is an open access article distributed under the Creative Commons Attribution License, which permits unrestricted use, distribution, and reproduction in any medium, provided the original work is properly cited.

Core and satellite structure is one of the common structures in enterprise clusters. In core and satellite structure, there are one core enterprise and at least two satellite enterprises. There exist a competitive relationship between satellite enterprises and a cooperative relationship between satellite enterprise and core enterprise. However, the dynamic evolution of competition-cooperation enterprise clusters with core-satellite structure is not well understood. In this paper, a novel competition-cooperation enterprise cluster model with core-satellite structure is proposed. The boundedness of the positive equilibrium is investigated. It is found that there exists upper bound of both core enterprise output and satellite enterprise output and the upper bound of core enterprise not only depends on its own production capacity but also depends on the production capacity of two satellite enterprises. Then, by selecting the production period as bifurcating parameter, the conditions of local stability and Hopf bifurcation are obtained. Once the production period passes a critical value, the output of both core enterprise and satellite enterprise loses stability and displays periodic fluctuations. This may lead to the decline of efficiency of enterprise and resource mismatch. Furthermore, the fluctuation properties are studied. Finally, a numerical example is presented to show the effectiveness of theorem.

\section{Introduction}

Recently, enterprise cluster, as an effective form of industrial space organization, has gradually become a common phenomenon in the process of modern industry and internationalization [1-4]. The competition-cooperation relationship widely exists in the real enterprise clusters and has a major impact on the evolution of enterprise clusters [5-9]. To study the influence of competition and cooperation on the evolution of enterprise clusters, researchers propose a competition-cooperation enterprise cluster model [10] based on the ecology model [11-15], which is described as follows:

$$
\left\{\begin{array}{l}
\dot{x}_{1}(t)=r_{1} x_{1}(t)\left(1-\frac{x_{1}(t)}{K_{1}}-\frac{\alpha\left(x_{2}(t-\tau)-c_{2}\right)^{2}}{K_{2}}\right), \\
\dot{x}_{2}(t)=r_{2} x_{2}(t)\left(1-\frac{x_{2}(t)}{K_{2}}+\frac{\alpha\left(x_{1}(t-\tau)-c_{1}\right)^{2}}{K_{1}}\right),
\end{array}\right.
$$


where $x_{1}, x_{2}$ are the enterprise output; $r_{i}$ is the intrinsic growth; $K_{i}(i=1,2)$ denotes the carrying capacity of market under nature unlimited conditions; $c_{i}(i=1,2)$ is the initial output of core enterprise; and $\tau$ is the production period. Let $a_{1}=\left(r_{1} / K\right), a_{2}=\left(r_{2} / K\right), b_{1}=\left(r_{1} \alpha / K\right), b_{2}=\left(r_{2} \beta / K\right)$, and system (1) can be rewritten as follows:

$$
\left\{\begin{array}{l}
\dot{x}_{1}(t)=x_{1}(t)\left(r_{1}-a_{1} x_{1}(t)-b_{1}\left(x_{2}(t-\tau)-d\right)^{2}\right), \\
\dot{x}_{2}(t)=x_{2}(t)\left(r_{2}-a_{2} x_{2}(t)+b_{2}\left(x_{1}(t-\tau)-d\right)^{2}\right) .
\end{array}\right.
$$

For this model, the dynamic behaviors including stability, Hopf bifurcation, and chaos have been widely studied [16-21].

In practical enterprise clusters, organization structure has a major impact on the production efficiency of enterprise clusters. The efficiency of overall operation is one of the important factors of enterprise's success. Thus, it is necessary to consider the structure in the enterprise cluster model. Among the many organization structures, core and satellite structure is one of the common structures in enterprise clusters, which is described in Figure 1. In core and satellite structure, there are one core enterprise and at least two satellite enterprises. There exist competitive relationship among satellite enterprises and cooperative relationship between satellite enterprise and core enterprise. For example, in automobile enterprise cluster, the core enterprise produces the motor vehicle and the satellite enterprise produces automobile parts for core enterprises. To reduce

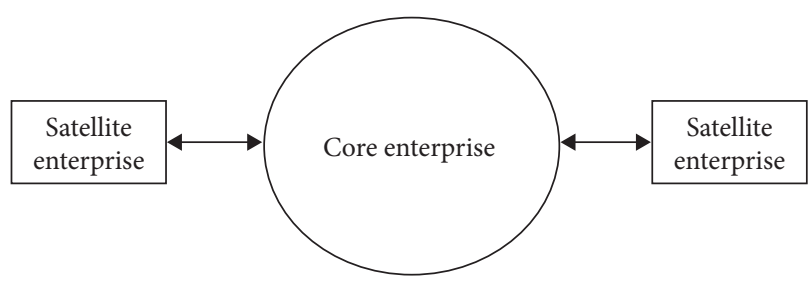

FIgURE 1: The enterprise cluster with one core enterprise and two satellite enterprises.

the cost and ensure the stability of supply chain, the core enterprise has at least two satellite enterprises for the same automobile part. It is easy to see that there exists competition between the two satellite enterprises and there exists cooperation between satellite enterprise and core enterprise. However, few works investigate the dynamic evolution of enterprise cluster model with core-satellite structure.

Inspired by the discussion, in this paper, a competitioncooperation enterprise cluster model composed of a core enterprise and two satellite enterprises is proposed, which is shown in Figure 1. In this enterprise cluster, there are one core enterprise and two satellite enterprises. There is a competitive relationship between two satellite enterprises. And there is a cooperative relationship between satellite enterprise and core enterprise. The model is described as follows:

$$
\left\{\begin{array}{l}
\dot{x}_{1}(t)=x_{1}(t)\left(r_{1}-a_{1} x_{1}(t)-b_{1} x_{2}(t)-c_{1}(y(t-\tau)-d)^{2}\right), \\
\dot{x}_{2}(t)=x_{2}(t)\left(r_{2}-a_{2} x_{2}(t)-b_{2} x_{1}(t)-c_{1}(y(t-\tau)-d)^{2}\right), \\
\dot{y}(t)=y(t)\left(r_{3}-a_{3} y(t)+c_{2}\left(\left(x_{1}(t-\tau)-d_{1}\right)^{2}+\left(x_{2}(t-\tau)-d_{2}\right)^{2}\right)\right),
\end{array}\right.
$$

where $x_{i}(t)$ is the satellite enterprise output; $y(t)$ is the core enterprise output; $a_{i}$ is the self-regulation of enterprise $i ; r_{i}$ is the intrinsic growth; $b_{i}$ is the completion rate of satellite enterprise; $c_{1}$ is the completion rate between satellite enterprise and core enterprise; $c_{2}$ is the rate of conversion of commodity into the reproduction of enterprise; $d$ is the initial output of core enterprise; $d_{1}$ is the initial output of satellite enterprise $x_{1} ; d_{2}$ is the initial output of satellite enterprise $x_{2}$; and $\tau$ is the production period. The main contributions of this paper are as follows:

(1) A competition-cooperation enterprise cluster model is composed of a core enterprise and two satellite enterprises. There is a competitive relationship between two satellite enterprises. And there is a cooperative relationship between satellite enterprise and core enterprise.

(2) The boundedness of positive equilibrium is investigated. And there exists a upper limit of output of enterprise cluster model.
(3) The production period plays a key role in dynamics of the proposed enterprise cluster. When it passes a critical value, the output of the enterprise cluster system loses its stability and displays a periodic fluctuation, which may cause a drop in productivity of the enterprise cluster system.

The remainder of this paper is organized as follows. In Section 2, the boundedness analysis of positive equilibrium is given. In Section 3, the conditions of Hopf bifurcation are discussed. In Section 4, the normal form of Hopf bifurcation is given. In Section 5, an example is given to verify the theoretical analysis. In Section 6, we give the economic meaning.

\section{Boundedness of Positive Equilibrium}

In this section, we investigate the boundedness of positive equilibrium. It can be seen that system (3) has more than three equilibria if any one of the enterprise output is zero. As it has no economic sense if one of the enterprise output is zero, we only study the property of positive equilibrium 
where all enterprise outputs are positive. Let $E^{*}=\left(x_{1}^{*}, x_{2}^{*}, y^{*}\right)$ be the positive equilibrium of system (3), where

$$
\begin{aligned}
& x_{1}^{*}=\frac{\left(a_{2} r_{1}-b_{1} r_{2}\right)-\left(a_{2} c_{1}-b_{1} c_{2}\right)\left(y^{*}-d\right)^{2}}{a_{1} a_{2}-b_{1} b_{2}}, \\
& x_{2}^{*}=\frac{\left(b_{2} r_{1}-a_{1} r_{2}\right)-\left(b_{2} c_{1}-a_{1} c_{2}\right)\left(y^{*}-d\right)^{2}}{b_{1} b_{2}-a_{1} a_{2}}, \\
& y^{*}=\frac{r_{3}+c_{2}\left(\left(x_{1}^{*}-d_{1}\right)^{2}+\left(x_{2}^{*}-d_{2}\right)^{2}\right)}{a_{3}} .
\end{aligned}
$$

From the perspective of enterprise management, the output of enterprise cannot be negative. Thus, the initial condition of enterprise output must satisfy $x_{1}(\theta) \geq 0$, $x_{2}(\theta) \geq 0$, and $y(\theta) \geq 0$ for $\theta \in[-\tau, 0]$. In respect of the boundedness of $E^{*}=\left(x_{1}^{*}, x_{2}^{*}, y^{*}\right)$, we have the following lemma.

Lemma 1 (see [21]). Let $c>0, d>0$.

(1) If $(d x(t) / d t) \geq x(d-c x)$ is satisfied, then $\lim _{t \rightarrow \infty}$ $\inf x(t) \geq(d / c)$ for all $t \geq 0$ and $x(0)>0$.

(2) If $(d x(t) / d t) \leq x(d-c x)$ is satisfied, then $\lim _{t \rightarrow \infty}$ $\sup x(t) \leq(d / c)$ for all $t \geq 0$ and $x(0)>0$.

\section{Theorem 1}

(1) Suppose $x_{i}(\theta) \geq 0,(i=1,2), \theta \in[-\tau, 0]$ and $x_{i}(0)$ $>0,(i=1,2), t>0$; there exists $\psi_{i}=\psi_{i}\left(x_{i}(\theta)\right)>$ $0,(i=1,2)$ such that $x_{i}(t)<\psi_{i},(i=1,2)$, where $\psi_{1}=$ $\max \left\{\sup _{-\tau<\theta<0} x \leq \theta \leq 0_{1}(\theta),\left(r_{1} / a_{1}\right)\right\}, \quad \psi_{2}=\max$ $\left\{\sup _{-\tau \leq \theta \leq 0} x_{2}(\theta),\left(r_{2} / a_{2}\right)\right\}$.

(2) Suppose $y(\theta) \geq 0, \theta \in[-\tau, 0]$ and $y(0)>0, t>0$; there exists $\psi_{3}=\psi_{3}(y(\theta))>0$, such that $y(t)<\psi_{3}$, where $\quad \psi_{3}=\max \left\{\sup _{-\tau \leq \theta \leq 0} y \quad(\theta), \min \left\{r_{1}-a_{1}\right.\right.$ $\left.\left.\psi, r_{2}-a_{2} \psi_{2}\right\}\right\}$
Proof. First, we investigate the boundedness of $x_{1}(t)$ and $x_{2}(t)$. By (3), one can obtain $\dot{x}_{1}(t) \geq 0$ for all $t>T$. Then, we have

$$
\frac{\mathrm{d} x_{1}(t)}{\mathrm{d} t} \leq x_{1}(t)\left(r_{1}-a_{1} x_{1}\right) .
$$

According to Lemma 1, one has

$$
\lim _{t \longrightarrow \infty} \sup x_{1} \leq \frac{r_{1}}{a_{1}} .
$$

Similarly, one has

$$
\lim _{t \longrightarrow \infty} \sup x_{2} \leq \frac{r_{2}}{a_{2}} .
$$

If there exists $\left\{t_{n}\right\}_{n=1}^{\infty}$ such that $\dot{x}_{1}\left(t_{n}\right) \geq 0$ is local max, where $x_{1}(t)<x_{1}\left(t_{n}\right) t \in\left(0, t_{n}\right)$, using the same method, one can obtain that $x_{1}(t)$ has upper limit at $t=t_{n}$. Thus, one has $\psi_{1}=\max \left\{\sup _{-\tau<\theta<0} x_{1}(\theta),\left(r_{1} / a_{1}\right)\right\}$. Similarly, we also can obtain $\psi_{2}=\max \left\{\sup _{-\tau \leq \theta \leq 0} x_{2}(\theta),\left(r_{2} / a_{2}\right)\right\}$.

In this same way, it follows that there exists $\psi_{3}>0$, such that $y(0)<\psi_{3}$ for $t>0$, where $\psi_{3}=\max \left\{\sup _{-\tau<\theta<0} y\right.$ $\left.(\theta), \min \left\{r_{1}-a_{1} \psi, r_{2}-a_{2} \psi_{2}\right\}\right\}$. We complete the proof.

Remark 1. From Theorem 1, one can see that there are upper bounds on the output of core enterprise and two satellite enterprises. Moreover, the upper bound of core enterprise not only depends on its own production capacity but also depends on the production capacity of two satellite enterprises.

\section{Bifurcation Analysis}

In this section, the conditions of Hopf bifurcation for (3) with $E^{*}=\left(x_{1}^{*}, x_{2}^{*}, y^{*}\right)$ are presented. By (3), one can obtain the Jacobian matrix for (3) with $E^{*}$ :

$$
\left(\begin{array}{ccc}
-a_{1} x_{1}^{*} & -b_{1} x_{1}^{*} & -2 c_{1} x_{1}^{*}\left(y^{*}-d\right) e^{-\lambda \tau} \\
-b_{2} x_{2}^{*} & -a_{2} x_{2}^{*} & -2 c_{1} x_{2}^{*}\left(y^{*}-d\right) e^{-\lambda \tau} \\
-2 c_{2} y^{*}\left(x_{1}^{*}-d_{1}\right) e^{-\lambda \tau} & -2 c_{2} y^{*}\left(x_{2}^{*}-d_{2}\right) e^{-\lambda \tau} & -a_{3} y^{*}
\end{array}\right) .
$$

Then, we have

where

$$
\lambda^{3}+e_{1} \lambda^{2}+e_{2} \lambda+e_{3}+e^{-2 \lambda \tau}\left(e_{4} \lambda+e_{5}\right)=0,
$$

$$
\begin{aligned}
& e_{1}=a_{1} x_{1}^{*}+a_{2} x_{2}^{*}+a_{3} x_{3}^{*}, \\
& e_{2}=a_{3} y^{*}\left(a_{2} x_{2}^{*}+a_{1} x_{1}^{*}\right)+x_{1}^{*} x_{2}^{*}\left(a_{1} a_{2}-b_{1} b_{2}\right), \\
& e_{3}=-4 c_{1} c_{2} y^{*}\left(y^{*}-d_{1}\right)\left[x_{1}^{*}\left(x_{1}^{*}-d_{1}\right)-x_{2}^{*}\left(x_{2}^{*}-d_{1}\right)\right], \\
& e_{4}=4 c_{1} c_{2} x_{1}^{*} x_{2}^{*} y^{*}\left(x_{1}^{*}-d_{1}\right)\left(y^{*}-d_{1}\right)\left(b_{1}-a_{1}\right)+4 c_{1} c_{2} x_{1}^{*} x_{2}^{*} y^{*}\left(x_{2}^{*}-d_{1}\right)\left(y^{*}-d_{1}\right)\left(b_{2}-a_{1}\right), \\
& e_{5}=a_{3} x_{1}^{*} x_{2}^{*} y^{*}\left(a_{1} a_{2}-b_{1} b_{2}\right) .
\end{aligned}
$$


If $i \omega(\omega>0)$ is a root of $(9)$, one can obtain

$-i \omega^{3}-e_{1} \omega^{2}+e_{2} i \omega+e_{3} i \omega e^{-2 i \omega \tau}+e_{4} e^{-2 i \omega \tau}+e_{5}=0$.

Separating the real and imaginary parts of (11), we have

$$
\begin{aligned}
& e_{3} \omega \sin 2 \omega \tau+e_{4} \cos 2 \omega \tau=e_{1} \omega^{2}-e_{5}, \\
& e_{3} \omega \cos 2 \omega \tau-e_{4} \sin 2 \omega \tau=\omega^{3}-e_{2} \omega .
\end{aligned}
$$

By (12) and (13), one can obtain

$$
\omega^{6}+f_{1} \omega^{4}+f_{2} \omega^{2}+f_{3}=0,
$$

where $f_{1}=e_{1}^{2}-2 e_{2}, f_{2}=e_{2}-2 e_{1} e_{5}-e_{3}^{2}, f_{3}=e_{5}^{2}-e_{4}^{2}$.

Let $z=\omega^{2}$; then, (14) becomes

$$
z^{3}+f_{1} z^{2}+f_{2} z+f_{3}=0
$$

Letting $z^{*}=(1 / 3)\left(-f_{1}+\sqrt{f_{1}^{2}-3 f_{2}}\right), h\left(z^{*}\right)=\left(z^{*}\right)^{3}+$ $f_{1}\left(z^{*}\right)^{2}+f_{2} z^{*}+f_{3}$, we get the following.

Lemma 2 (see [22-24]).

(i) If $f_{3}<0$, (15) has at least one positive root.

(ii) If $f_{3} \geq 0$ and $f_{1}^{2}-f c_{2} \leq 0$, (15) has no positive root.

(iii) If $f_{3} \geq 0, f_{1}^{2}-3 f_{2}>0, z^{*}>0$, and $h\left(z^{*}\right) \leq 0$, (15) has positive roots.
Assume (14) has three positive roots $\omega_{k}=\sqrt{z_{k}}, k=1,2$, 3. By (12) and (13), we have

$$
\cos 2 \omega \tau=\frac{e_{3} \omega^{4}+e_{1} e_{4} \omega^{2}-e_{2} e_{3} \omega^{2}+e_{4} e_{5}}{e_{3} \omega^{2}+e_{4}^{2}} .
$$

Thus, denote

$$
\tau_{k}^{j}=\frac{1}{2 \omega_{k}} \operatorname{arcos} \frac{e_{3} \omega^{4}+e_{1} e_{4} \omega^{2}-e_{2} e_{3} \omega^{2}+e_{4} e_{5}}{e_{3} \omega^{2}+e_{4}^{2}}+\frac{2 j \pi}{2 \omega_{k}},
$$

where $k=1,2,3 ; j=0,2, \quad \ldots$ Define $\tau_{0}=\tau_{k_{0}}^{0}=$ $\min _{k=1,2,3}\left\{\tau_{k_{0}}^{0}\right\}, \omega_{0}=\omega_{k_{0}}$.

Note that when $\tau=0$, (9) becomes

$$
\lambda^{3}+e_{1} \lambda^{2}+\left(e_{2}+e_{3}\right) \lambda+e_{4}+e_{5}=0 .
$$

By using the Routh-Hurwitz criterion [16], one can obtain the condition that all roots of (18) have negative real parts.

$$
(\mathrm{H} 1) e_{1}\left(e_{2}+e_{4}\right)-\left(e_{3}+e_{5}\right)>0 \text {. }
$$

Lemma 3 (see [25]). Consider the exponential polynomial

$$
\begin{aligned}
P\left(\lambda, e^{-\lambda \tau}, \ldots \ldots, e^{-\lambda \tau_{m}}\right)= & \lambda^{n}+p_{1}^{(0)} \lambda^{n-1}+\ldots \ldots+p_{n-1}^{(0)} \lambda+p_{n}^{(0)}+\left[p_{1}^{(1)} \lambda^{n-1}+\ldots \ldots+p_{n-1}^{(1)} \lambda+p_{n}^{(1)}\right] e^{-\lambda \tau} \\
& +\ldots \ldots+\left[p_{1}^{(m)} \lambda^{n-1}+\ldots \ldots+p_{n-1}^{(m)} \lambda+p_{n}^{(m)}\right] e^{-\lambda \tau_{m}}
\end{aligned}
$$

where $\tau_{i} \geq 0(i=1,2, \ldots, m)$ and $\left(p_{j}^{(i)}=(j=1,2, \ldots, m)\right.$ are constants. As $\left(\tau_{1}, \tau_{2}, \ldots, \tau_{m}\right)$ vary, the sum of the order of the zeros of $P\left(\lambda, e^{-\lambda \tau}, \ldots, e^{-\lambda \tau_{m}}\right)$ on the open right half plane can change only if a zero appears on or crosses the imaginary axis.

Lemma 4. If $h^{\prime}\left(z_{0}\right) \neq 0$, then $\operatorname{sgn}\left\{\operatorname{Re}\left\{((d \lambda / d \tau))^{-1}\right\}\right\} \neq 0$ when $\tau=\tau_{0}$ and the sign of $h^{\prime}\left(z_{0}\right)$ is same as the sign of $\left.\operatorname{Re}\{((d \lambda / d \tau))\}\right|_{\tau=\tau_{0}}$.

Proof. Differentiating (9) with respect to $\tau$ yields

$$
\left\{3 \lambda^{2}+2 e_{1} \lambda+e_{2}+\left[e_{3}-2 \tau\left(e_{3} \lambda+e_{4}\right)\right] e^{-2 \lambda \tau}\right\} \frac{\mathrm{d} \lambda}{\mathrm{d} \tau}=2 \lambda\left(e_{3} \lambda+e_{4}\right) e^{-2 \lambda \tau} .
$$

For convenience, we denote $\omega_{0}$ and $\tau_{0}$ by $\omega$ and $\tau$; then, we have

$$
\left(\frac{\mathrm{d} \lambda}{\mathrm{d} \tau}\right)^{-1}=\frac{\left(3 \lambda^{2}+2 e_{1} \lambda+e_{2}\right) e^{2 \lambda \tau}}{2 \lambda\left(e_{3} \lambda+e_{4}\right)}+\frac{e_{3}}{2 \lambda\left(e_{3} \lambda+e_{4}\right)}-\frac{\tau}{\lambda} .
$$

Then, we get

$$
\begin{aligned}
& \operatorname{Re}\left\{\left(\frac{\mathrm{d} \lambda}{\mathrm{d} \tau}\right)^{-1}\right\}_{\tau=\tau_{0}} \\
& =\operatorname{Re}\left\{\frac{\left(3 \lambda^{2}+2 e_{1} \lambda+e_{2}\right) e^{2 \lambda \tau}}{2 \lambda\left(e_{3} \lambda+e_{4}\right)}\right\}_{\tau=\tau_{0}}+\operatorname{Re}\left\{\frac{e_{3}}{2 \lambda\left(e_{3} \lambda+e_{4}\right)}\right\}_{\tau==_{\tau}^{0}} \\
& =\frac{1}{A_{k}}\left[-2 \omega_{0}\left(e_{2}-3 \omega_{0}^{2}\right)\left(\omega_{0}^{3}-e_{2} \omega_{0}\right)+4 e_{1} \omega_{0}^{2}\left(e_{1} \omega_{0}^{2}-e_{5}\right)-2 e_{3}^{2} \omega_{0}^{2}\right] \\
& =\frac{1}{A_{k}}\left(6 \omega_{0}^{6}+4 f \omega_{0}^{4}+2 f_{2} \omega_{0}^{2}\right) \\
& =\frac{2}{A_{k}}\left[z_{0}\left(3 z_{0}^{2}+2 f_{1} z_{0}+f_{2}\right)\right] \\
& =\frac{2 z_{0}}{A_{k}} h^{\prime}\left(z_{0}\right),
\end{aligned}
$$

where $A_{k}=4 e_{3}^{2} \omega_{0}^{4}+4 e_{4}^{2} \omega_{0}^{2}>0$.

Then, if $h^{\prime}\left(z_{0}\right) \neq 0$, we have $\operatorname{sgn}\left\{\operatorname{Re}\left\{((\mathrm{d} \lambda / \mathrm{d} \tau))^{-1}\right\}\right\} \neq 0$, and the sign of $h^{\prime}\left(z_{0}\right)$ is the same as the sign of $\left.\operatorname{Re}\{(d \lambda / d \tau)\}\right|_{\tau=\tau_{0}}$. We complete the proof.

Thus, from Lemmas 2, 3 , and 4 , one has the following. 
Theorem 2. Suppose that (H1) holds, and we have

(1) If $f_{3}>0$ and $f_{1}^{2}-3 f_{2} \leq 0$, (3) is asymptotically stable at $E^{*}$.

(2) If either $f_{3}<0$ or $f_{3} \geq 0$ and $f_{1}^{2}-3 f_{2}>0 \quad z^{*}>0$, $h\left(z^{*}\right) \leq 0$ holds, (3) is asymptotically stable at $E^{*}$ for $\tau \in\left[0, \tau_{0}\right)$ and undergoes a Hopf bifurcation at $E^{*}$ when $\tau=\tau_{0}$.

\section{Direction of the Hopf Bifurcation}

In this section, we study the properties of Hopf bifurcation by using the center manifold [23, 26-28]. Letting $x_{1}=x_{1}-$ $x_{1}^{*}, x_{2}=x_{2}-x_{2}^{*}, x_{3}=x_{3}-x_{3}^{*}, \bar{x}_{i}(t)=x_{i}(\tau t), \tau=\tau_{0}+\mu,(3)$ can be transformed into a FDE as

$$
\dot{x}(t)=L_{\mu}\left(x_{t}\right)+f\left(\mu, x_{t}\right) \text {, }
$$

with

$$
L_{\mu} \phi=\left(\tau_{0}+\mu\right)\left[B_{1} \varphi(0)+B_{2} \varphi(-1)\right]
$$

where

$$
\begin{aligned}
B_{1} & =\left[\begin{array}{lll}
b_{11} & b_{12} & 0 \\
b_{21} & b_{22} & 0 \\
0 & 0 & b_{33}
\end{array}\right], \\
B_{2} & =\left[\begin{array}{ccc}
0 & 0 & b_{13} \\
0 & 0 & b_{23} \\
b_{31} & b_{32} & 0
\end{array}\right], \\
f(\mu, \varphi) & =\left(\tau_{0}+\mu\right)\left(\begin{array}{c}
f_{1} \\
f_{2} \\
f_{3}
\end{array}\right),
\end{aligned}
$$

where 


$$
\begin{aligned}
& b_{36}=-a_{3}, \\
& b_{37}=-2 c_{2} d_{1}, \\
& b_{38}=-2 c_{2} d_{2}, \\
& b_{39}=c_{2} .
\end{aligned}
$$

According to Riesz representation theorem, there exists a function $\eta(\theta, \mu)$ of bounded variation for $\theta \in[-1,0]$, such that

$$
L_{\mu} \varphi=\int_{-1}^{0} \mathrm{~d} \eta(\theta, \mu) \varphi(\theta), \quad \varphi \in C
$$

Let

$$
\eta(\theta, \mu)=\left(\tau_{0}+\mu\right)\left[B_{1} \delta(\theta)+B_{2} \delta(\theta+1)\right],
$$

where $\delta(\theta)$ is Dirac delta function.

By [21], we define

$$
\begin{aligned}
& A(\mu) \varphi= \begin{cases}\frac{\mathrm{d} \varphi}{\mathrm{d} \theta}, & \theta \in[-1,0), \\
\int_{-1}^{0} \mathrm{~d} \eta(\theta, \mu) \varphi(\theta), & \theta=0,\end{cases} \\
& R(\mu) \varphi= \begin{cases}0, & \theta \in[-1,0], \\
f(\mu, \varphi), & \theta=0 .\end{cases}
\end{aligned}
$$

Then, system (24) can be rewritten as

$$
\dot{x}(t)=A(\mu) x_{t}+R(\mu) x_{t},
$$

where $x_{t}(\theta)=x(t+\theta)$

The adjoint operator $A^{*}$ of $A$ is defined by

$$
A^{*}(\mu) \psi= \begin{cases}-\frac{\mathrm{d} \psi(s)}{\mathrm{d} \theta}, & s \in(0,1], \\ \int_{-1}^{0} \mathrm{~d} \eta^{T}(t, 0) \psi(-t), & s=0,\end{cases}
$$

where $\eta^{T}$ is the transpose of the matrix $\eta$.

For $\varphi \in C^{1}[-1,0]$ and $\psi \in C^{1}[0,1]$, we define

$$
\langle\psi, \varphi\rangle=\bar{\psi}(0) \cdot \varphi(0)-\int_{\theta=-1}^{0} \int_{\xi=0}^{\theta} \bar{\psi}(\xi-\theta) \mathrm{d} \eta(\theta) \varphi(\xi) \mathrm{d} \xi,
$$

where $\eta(\theta)=\eta(\theta, 0)$. We know that $\pm i \tau_{0} \omega_{0}$ is an eigenvalue of $A(0)$, so $\pm i \tau_{0} \omega_{0}$ is also an eigenvalue of $A^{*}(0)$. We can get

$$
q(\theta)=\left(\begin{array}{l}
1 \\
\alpha \\
\beta
\end{array}\right) e^{i \tau_{0} \omega_{0} \theta}, \quad-1<\theta \leq 0 .
$$

By [21], we have

$$
A q(0)=i \tau_{0} \omega_{0} q(0)
$$

Hence, one can obtain

$$
\begin{gathered}
\alpha=-\frac{b_{21}\left(i \omega_{0}+b_{33}\right)+b_{31} e^{-2 i \omega_{0} \tau_{0}}}{\left(i \omega_{0}+b_{22}\right)\left(i \omega_{0}+b_{33}\right)+b_{32} b_{23} e^{-2 i \omega_{0} \tau_{0}}}, \\
\beta=\frac{\left(b_{31}\left(i \omega_{0}+b_{22}\right)-b_{21} b_{32}\right) e^{-i \omega_{0} \tau_{0}}}{\left(i \omega_{0}+b_{22}\right)\left(i \omega_{0}+b_{33}\right)+b_{32} b_{23} e^{-2 i \omega_{0} \tau_{0}}} .
\end{gathered}
$$

Assume that the eigenvector $q^{*}$ of $A^{*}$ is

$$
q^{*}(s)=\left(\begin{array}{c}
1 \\
\alpha^{*} \\
\beta^{*}
\end{array}\right) e^{i \tau_{0} \omega_{0} s} .
$$

By [21], we have

$$
A^{*} q(0)=-i \tau_{0} \omega_{0} q^{*}(0) .
$$

Hence, we obtain

$$
\begin{aligned}
& \alpha^{*}=\frac{b_{13} b_{21}+b_{23}\left(i \omega_{0}-b_{11}\right)}{b_{12} b_{23} e^{-i \omega_{0} \tau_{0}}+b_{13}\left(i \omega_{0}-b_{22}\right)}, \\
& \beta^{*}=\frac{\left(\left(i \omega_{0}-b_{11}\right)\left(i \omega_{0}-b_{22}\right)-b_{12} b_{21}\right) e^{i \omega_{0} \tau_{0}}}{\left(i \omega_{0}-b_{22}\right)\left(b_{13}+b_{21}\right)} .
\end{aligned}
$$

Let

$$
\left\langle q^{*}, q\right\rangle=1 \text {. }
$$

Then, we can compute

$$
\begin{aligned}
\left\langle q^{*}, q\right\rangle & =q^{*}(0) \cdot q(0)-\int_{\theta=-1}^{0} \int_{\xi=0}^{\theta}-q^{*^{T}}(\xi-\theta) \mathrm{d} \eta(\theta) q(\xi) \mathrm{d} \xi \\
& =\frac{1}{\bar{\rho}}\left(1+\alpha \alpha^{*}+\beta \beta^{*}\right)-\int_{\theta=-1}^{0} \frac{\theta}{\bar{\rho}} \tau_{0} e^{i w_{0} \tau_{0} \theta}\left[\left(b_{11}+b_{21} \bar{\alpha}^{*}+b_{12} \alpha+b_{22} \alpha \bar{\alpha}^{*}+b_{33} \beta \bar{\beta}^{*}\right) \delta(\theta)\right.
\end{aligned}
$$




$$
\begin{aligned}
& \left.+\left(b_{31} \bar{\beta}^{*}+b_{32} \alpha \bar{\beta}^{*}+b_{13} \beta+b_{23} \bar{\alpha}^{*} \beta\right) \delta(\theta+1)\right] \mathrm{d} \theta \mathrm{d} \xi \\
= & \frac{1}{\bar{\rho}}\left(1+\alpha \alpha^{*}+\beta \beta^{*}\right)+\frac{\tau_{0}}{\bar{\rho}} e^{-i \tau_{0} \omega_{0}}\left(b_{31} \bar{\beta}^{*}+b_{32} \alpha \bar{\beta}^{*}+b_{13} \beta+b_{23} \bar{\beta}^{*} \beta\right) \\
= & 1 .
\end{aligned}
$$

Hence, we obtain

$$
\bar{\rho}=\left(1+\alpha \alpha^{*}+\beta \beta^{*}\right)+\tau_{0} e^{-i \tau_{0} \omega_{0}}\left(b_{31} \bar{\beta}^{*}+b_{32} \alpha \bar{\beta}^{*}+b_{13} \beta+b_{23} \bar{\alpha}^{*} \beta\right) \text {. }
$$

Assume that $x_{t}$ is a solution of (33) with $\mu=0$; we define

$$
\begin{aligned}
z(t) & =\left\langle q^{*}, x_{t}\right\rangle, \\
W(t, \theta) & =x_{t}-2 \operatorname{Re}\{z(t) q(\theta)\} .
\end{aligned}
$$

$$
W(t, \theta)=W(z, \bar{z}, t)
$$

where

$$
W(z, \bar{z}, t)=W_{20}(\theta) \frac{z^{2}}{2}+W_{11}(\theta) \frac{z \bar{z}}{2}+W_{02}(\theta) \frac{\bar{z}^{2}}{2}+\ldots
$$

In fact, $z$ and $\bar{z}$ are local coordinates for $C_{0}$ in $q$ and $q^{*}$. As $\mu=0$, we have

On $C_{0}$, one has

$$
\begin{aligned}
\dot{z}(t) & =\left\langle q^{*}, \dot{x}_{t}\right\rangle=\left\langle q^{*}, A(\mu) x_{t}+R(\mu) x_{t}\right\rangle=\left\langle q^{*}, A x_{t}\right\rangle+\left\langle q^{*}, R x_{t}\right\rangle \\
& =i \tau_{0} w_{0} z+\bar{q}^{*}(0) \cdot f(0, W(t, 0)+2 \operatorname{Re}[z(t) q(0)]) .
\end{aligned}
$$

Rewrite (39) as

$$
\dot{z}(t)=i \tau_{0} \omega_{0} z+g(z, \bar{z}),
$$

$$
g(z, \bar{z})=g_{20} \frac{z^{2}}{2}+g_{11} z \bar{z}+g_{02} \frac{\bar{z}^{2}}{2}+g_{21} \frac{z^{2} \bar{z}}{2}+\ldots
$$

By (37) and (40), one has

where

$$
\dot{W}=\dot{x}_{t}-\dot{z} q-\dot{\bar{z}} \dot{q}= \begin{cases}A W-2 \operatorname{Re}\left[\bar{q}^{*}(0) \cdot f(z, \bar{z}) q(\theta)\right], & \theta \in[-2 \tau, 0), \\ A W-2 \operatorname{Re}\left[\bar{q}^{*}(0) \cdot f(z, \bar{z}) q(\theta)\right]+f_{0}(z, \bar{z}), & \theta=0 .\end{cases}
$$

Let

$$
\dot{W}=A W+H(z, \bar{z}, \theta),
$$

where

$$
H(z, \bar{z}, \theta)=H_{20}(\theta) \frac{z^{2}}{2}+H_{11}(\theta) \frac{z \bar{z}}{2}+H_{02}(\theta) \frac{\bar{z}^{2}}{2}+\ldots
$$

Following the method in [18-21], one can obtain

$$
\begin{aligned}
\left(A-2 i w_{0}\right) W_{20}(\theta) & =-H_{20}(\theta), \\
A W_{11}(\theta) & =-H_{11}(\theta), \\
\left(A+2 i w_{0}\right) W_{02}(\theta) & =-H_{02}(\theta) .
\end{aligned}
$$

Since $x_{t}=x(t+\theta)=W(z, \bar{z}, \theta)+z q+\bar{z} \cdot \bar{q}$, one has

$$
x_{t}=\left(\begin{array}{c}
W^{(1)}(z, \bar{z}, \theta) \\
W^{(2)}(z, \bar{z}, \theta) \\
W^{(3)}(z, \bar{z}, \theta)
\end{array}\right)+z\left(\begin{array}{c}
1 \\
q_{1} \\
q_{2}
\end{array}\right) e^{i \omega_{0} \theta}+\bar{z}\left(\begin{array}{c}
1 \\
\bar{q}_{1} \\
\bar{q}_{2}
\end{array}\right) e^{-i \omega_{0} \theta} .
$$

Thus, one can obtain

$$
\varphi_{1}(0)=z+\bar{z}+W_{20}^{(1)}(0) \frac{z^{2}}{2}+W_{11}^{(1)}(0) z \bar{z}+W_{02}^{(1)}(0) \frac{\bar{z}^{2}}{2}
$$$$
\varphi_{2}(0)=z q_{1}+\bar{z} \bar{q}_{1}^{*}+W_{20}^{(1)}(0) \frac{z^{2}}{2}+W_{11}^{(1)}(0) z \bar{z}+W_{02}^{(1)}(0) \frac{\bar{z}^{2}}{2}
$$

So, 


$$
\varphi_{1}(0) \varphi_{2}(0)=q_{1} z^{2}+\bar{q}_{1} \bar{z}^{2}+\left(q_{1}+q_{2}\right) z \bar{z}+\left(W_{11}^{(2)}+\frac{1}{2} W_{20}^{(2)}+W_{11}^{(1)} q_{1}+\frac{1}{2} W_{20}^{(1)} \bar{q}\right) z^{2} \bar{z} .
$$

By (27) and (43), one can obtain where

$$
f(\varphi, \mu)=\left(\begin{array}{l}
K_{11} z^{2}+K_{12} z \bar{z}+K_{13} \bar{z}^{2} \\
K_{21} z^{2}+K_{22} z \bar{z}+K_{23} \bar{z}^{2} \\
K_{31} z^{2}+K_{32} z \bar{z}+K_{33} \bar{z}^{2}
\end{array}\right),
$$

$$
\begin{aligned}
& K_{11}=b_{14}+\alpha b_{15}+b_{16} \beta^{2} e^{-2 i \omega_{0} \tau_{0}}, \\
& K_{12}=2 b_{14}+b_{15} \alpha+b_{15} \bar{\alpha}+b_{17} \beta e^{-i \omega_{0} \tau_{0}}+2 \beta \bar{\beta} b_{16}+\bar{\beta} b_{17} e^{i \omega_{0} \tau_{0}}, \\
& K_{13}=b_{14}+\bar{\alpha} b_{15}+b_{16} \bar{\beta}^{2} e^{2 i \omega_{0} \tau_{0}}+b_{17} \bar{\beta} e^{i \omega_{0} \tau_{0}}, \\
& K_{14}=2 b_{14} W_{11}^{(1)}(0)+b_{15} W_{11}^{(2)}(0)+b_{14} W_{20}^{(1)}(0)+\frac{1}{2} b_{15} W_{20}^{(2)}(0)+b_{17} W_{11}^{(3)}(-1) \\
& +\frac{1}{2} b_{17} W_{20}^{(3)}(-1)+\beta^{2} b_{18} e^{-2 i \omega_{0} \tau_{0}}+2 \beta \bar{\beta} b_{18}+b_{15} \alpha W_{11}^{(1)}(0)+b_{17} \beta W_{11}^{(1)}(0) \\
& +2 \beta b_{16} e^{-i \omega_{0} \tau_{0}} W_{11}^{(3)}(-1)+\frac{1}{2} \bar{\beta} b_{17} W_{20}^{(1)}(0) e^{i \omega_{0} \tau_{0}}+\bar{\beta} b_{16} W_{20}^{(3)}(-1) e^{i \omega_{0} \tau_{0}}, \\
& K_{21}=b_{25} \alpha^{2}+b_{27} \beta \alpha e^{-i \omega_{0} \tau_{0}}+b_{24} \alpha+b_{26} \beta^{2} e^{-2 i \omega_{0} \tau_{0}}, \\
& K_{22}=2 b_{25} \alpha \bar{\alpha}+b_{27} \alpha \bar{\beta} e^{i \omega_{0} \tau_{0}}+b_{24} \alpha+b_{27} \bar{\alpha} \beta e^{-i \omega_{0} \tau_{0}}+b_{24} \bar{\alpha}+2 b_{26} \beta \bar{\beta}, \\
& K_{23}=b_{25} \bar{\alpha}^{2}+b_{27} e^{i \omega_{0} \tau_{0}} \bar{\alpha} \bar{\beta}+b_{24} \bar{\alpha}+b_{26} \bar{\beta}^{2} e^{2 i \omega_{0} \tau_{0}}, \\
& K_{24}=2 b_{28} \alpha \beta \bar{\beta}+2 b_{5} \alpha W_{11}^{(2)}(0)+b_{27} \alpha W_{11}^{(3)}(-1)+b_{24} W_{11}^{(1)}(0) \alpha+b_{28} \bar{\alpha} \beta^{2} e^{-2 i \omega_{0} \tau_{0}} \\
& +b_{25} \bar{\alpha} W_{20}^{(2)}(0)+\frac{1}{2} b_{27} \bar{\alpha} W_{20}^{(3)}(-1)+\frac{1}{2} b_{24} \bar{\alpha} W_{20}^{(1)}(0)+2 b_{26} e^{-i \omega_{0} \tau_{0}} W_{11}^{(3)}(-1) \\
& +\frac{1}{2} b_{27} e^{i \omega_{0} \tau_{0}} \bar{\beta} W_{20}^{(2)}(0)+b_{26} \bar{\beta} W_{20}^{(3)}(-1) e^{-i \omega_{0} \tau_{0}}+b_{24} W_{11}^{(2)}(0)+\frac{1}{2} b_{24} W_{20}^{(2)}(0), \\
& K_{31}=b_{35} e^{-2 i w_{0} \tau_{0}} \alpha^{2}+b_{38} e^{-i w_{0} \tau_{0}} \alpha \beta+b_{36} \beta^{2}+b_{37} e^{-i w_{0} \tau_{0}} \beta+b_{34} e^{-2 i w_{0} \tau_{0}}, \\
& K_{32}=2 b_{35} \alpha \bar{\alpha}+b_{38} \alpha \bar{\beta} e^{-i w_{0} \tau_{0}}+b_{38} \bar{\alpha} \beta e^{i w_{0} \tau_{0}}+2 b_{36} \beta \bar{\beta}+b_{37} \beta e^{i w_{0} \tau_{0}}+2 b_{34}, \\
& K_{33}=b_{35} \bar{\alpha}^{2} e^{i w_{0} \tau_{0}}+b_{38} \bar{\alpha} \bar{\beta} e^{i w_{0} \tau_{0}}+b_{36} \bar{\beta}^{2}+b_{37} \bar{\beta} e^{i w_{0} \tau_{0}}+b_{34} e^{2 i w_{0} \tau_{0}}, \\
& K_{34}=b_{39} \alpha^{2} \beta e^{-2 i w_{0} \tau_{0}}+2 b_{39} \alpha \bar{\alpha} \beta+b_{38} \alpha W_{02}^{(2)}(-1) e^{-i w_{0} \tau_{0}}+2 b_{35} e^{-i w_{0} \tau_{0}} \alpha W_{11}^{(2)}(-1) \\
& +b_{35} \alpha W_{20}^{(2)}(-1) e^{i w_{0} \tau_{0}}+2 b_{36} \beta W_{11}^{(3)}(0)+b_{37} \beta W_{11}^{(1)}(-1)+b_{38} \beta W_{11}^{(2)}(-1) \\
& +2 b_{39} \beta+b_{39} \bar{\beta} e^{-2 i w_{0} \tau_{0}}+b_{37} W_{11}^{(3)}(0) e^{-i w_{0} \tau_{0}}+\frac{1}{2} b_{37} W_{20}^{(3)} e^{i w_{0} \tau_{0}} \\
& ++2 b_{34} W_{11}^{(1)}(-1) e^{-i w_{0} \tau_{0}}+b_{34} W_{20}^{(1)}(-1) e^{i w_{0} \tau_{0}} .
\end{aligned}
$$


As $q^{*}(0)=(1 / \rho)\left(1, \bar{q}_{1}^{*}, \bar{q}_{2}^{*}\right)^{T}$, one has

$$
g(z, \bar{z})=\frac{1}{\rho}\left(1, \bar{q}_{1}^{*}, \bar{q}_{2}^{*}\right)\left(\begin{array}{c}
K_{11} z^{2}+K_{12} z \bar{z}+K_{13} \bar{z}^{2} \\
K_{21} z^{2}+K_{22} z \bar{z}+K_{23} \bar{z}^{2} \\
K_{31} z^{2}+K_{32} z \bar{z}+K_{33} \bar{z}^{2}
\end{array}\right) .
$$

Then, we have

$$
\begin{aligned}
& g_{20}=\frac{2}{\rho}\left(K_{11}+\bar{\alpha}^{*} K_{21}+\bar{\beta}^{*} K_{31}\right), \\
& g_{11}=\frac{1}{\rho}\left(K_{12}+\bar{\alpha}^{*} K_{22}+\bar{\beta}^{*} K_{32}\right), \\
& g_{02}=\frac{2}{\rho}\left(K_{13}+\bar{\alpha}^{*} K_{23}+\bar{\beta}^{*} K_{33}\right) .
\end{aligned}
$$

Next, we compute $W_{20}(\theta)$ and $W_{11}(\theta)$. According to the expression of $g_{21}$, we have $H(z, \bar{z}, \theta)=-2 \operatorname{Re}$ $\left[\bar{q}^{*}(0) \cdot f(z, \bar{z}) q(\theta)\right]$.

$$
=-\left(g_{20} \frac{z^{2}}{2}+g_{11} z \bar{z}+g_{02} \frac{\bar{z}^{2}}{2}+\ldots\right) q(\theta)-\left(\bar{g}_{20} \frac{\bar{z}^{2}}{2}+\bar{g}_{11} z \bar{z}+\bar{g}_{02} \frac{z^{2}}{2}+\ldots\right) q(\theta)
$$

Comparing the coefficients of (63), one has

$$
\begin{array}{ll}
H_{20}(\theta)=-g_{20} q(\theta)-\bar{g}_{02} \bar{q}(\theta), & \theta \in[-1,0), \\
H_{11}(\theta)=-g_{11} q(\theta)-\bar{g}_{11} \bar{q}(\theta), & \theta \in[-1,0) .
\end{array}
$$

Substituting (64) and (65) into (55), one can obtain

$$
\left\{\begin{array}{l}
\dot{W}_{20}(\theta)=2 i \tau_{0} \omega_{0} W_{20}(\theta)+g_{20} q(\theta)+\bar{g}_{20} \bar{q}(\theta) \\
\dot{W}_{11}(\theta)=+g_{11} q(\theta)+\bar{g}_{11} \bar{q}(\theta)
\end{array}\right.
$$

So,

$$
\left\{\begin{array}{l}
W_{20}(\theta)=\frac{i g_{20}}{\tau_{0} \omega_{0}} q(0) e^{i \tau_{0} \omega_{0} \theta}-\frac{\bar{g}_{02}}{3 i \tau_{0} \omega_{0}} \bar{q}(0) e^{-i \tau_{0} \omega_{0} \theta}+E_{1} e^{2 i \tau_{0} \omega_{0} \theta}, \\
W_{11}(\theta)=\frac{g_{11}}{i \tau_{0} \omega_{0}} q(0) e^{i \tau_{0} \omega_{0} \theta}-\frac{\bar{g}_{11}}{i \tau_{0} \omega_{0}} \bar{q}(0) e^{-i \tau_{0} \omega_{0} \theta}+E_{2} .
\end{array}\right.
$$

Now, we compute $E_{1}$ and $E_{2}$. From the definition of $A$ in (31), one can obtain

$$
\begin{aligned}
& \int_{-1}^{0} d \eta(\theta) W_{20}(\theta)=2 i \tau_{0} \omega_{0} W_{20}(0)-H_{20}(0), \\
& \int_{-1}^{0} d \eta(\theta) W_{11}(\theta)=-H_{11}(0) .
\end{aligned}
$$

From (50), (68), and (69), we have

$$
\begin{aligned}
& H_{20}(\theta)=-g_{20} q(\theta)-\bar{g}_{02} \bar{q}(\theta)+\left(K_{11}, K_{21}, 0\right)^{T}, \\
& H_{11}(\theta)=-g_{11} q(\theta)-\bar{g}_{11} \bar{q}(\theta)+\left(K_{12}, K_{22}, 0\right)^{T} .
\end{aligned}
$$

Following the method in [18-21], we have

$$
\begin{array}{r}
\left(i \omega_{0} I-\int_{-1}^{0} e^{i \omega_{0} \theta} d \eta(\theta)\right) q(0)=0 \\
\left(-i \omega_{0} I-\int_{-1}^{0} e^{-i \omega_{0} \theta} \mathrm{d} \eta(\theta)\right) \bar{q}(0)=0 .
\end{array}
$$

Then, we can obtain

$$
\left(2 i \omega_{0} I-\int_{-1}^{0} e^{2 i \tau_{0} \omega_{0} \theta} \mathrm{d} \eta(\theta)\right) E_{1}=\left(\begin{array}{lll}
K_{11} & K_{21} & 0
\end{array}\right)^{T},
$$

which leads to

$$
\left(\begin{array}{ccc}
2 i \omega_{0}+b_{11} & b_{12} & b_{13} e^{-i \omega_{0} \tau_{0}} \\
b_{21} & 2 i \omega_{0}+b_{22} & b_{23} e^{-i \omega_{0} \tau_{0}} \\
-b_{31} e^{-i \omega_{0} \tau_{0}} & -b_{32} e^{-i \omega_{0} \tau_{0}} & 2 i \omega_{0}+b_{33}
\end{array}\right) E_{1}=\left(\begin{array}{c}
K_{11} \\
K_{21} \\
K_{31}
\end{array}\right)
$$

and

$$
\left(\begin{array}{ccc}
-b_{11} & -b_{12} & -b_{13} \\
-b_{21} & -b_{22} & -b_{23} \\
b_{31} & b_{32} & -b_{33}
\end{array}\right) E_{2}=\left(\begin{array}{c}
K_{12} \\
K_{22} \\
K_{32}
\end{array}\right)
$$

Then, by (72) and (74), we can obtain the following properties of Hopf bifurcation:

$$
\begin{aligned}
& \mu_{2}=-\frac{\operatorname{Re} C_{1}(0)}{\operatorname{Re} \lambda^{\prime}\left(\tau_{0}\right)}, \\
& T_{2}=-\frac{\operatorname{Im} C_{1}(0)+\mu_{2} \operatorname{Im} \lambda^{\prime}(0)}{\omega_{0}},
\end{aligned}
$$

$\beta_{2}=-2 \operatorname{ReC}_{1}(0)$ 

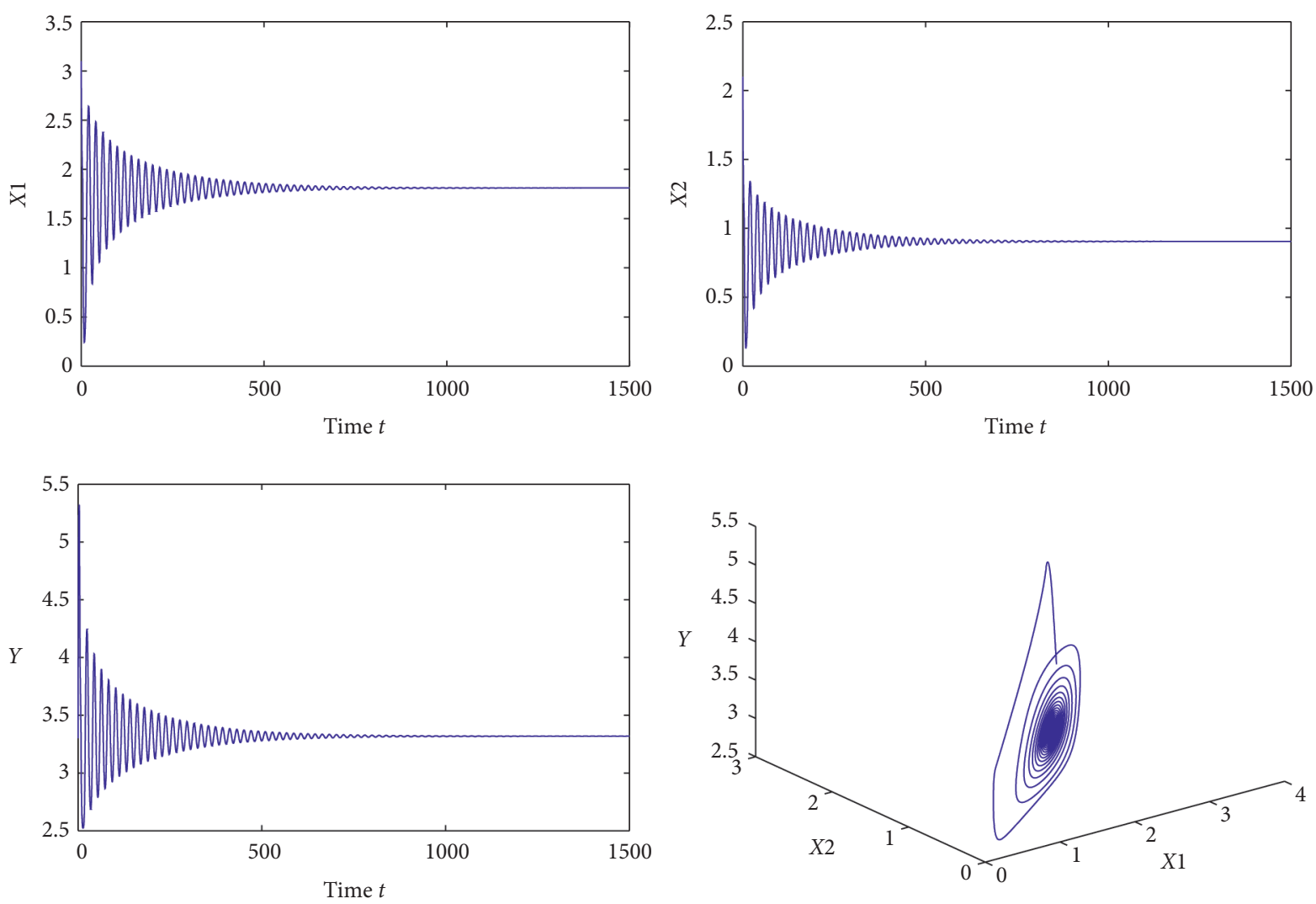

FIgURE 2: $\tau=4.05<\tau_{0}$. The positive equilibrium of system (3) is asymptotically stable.
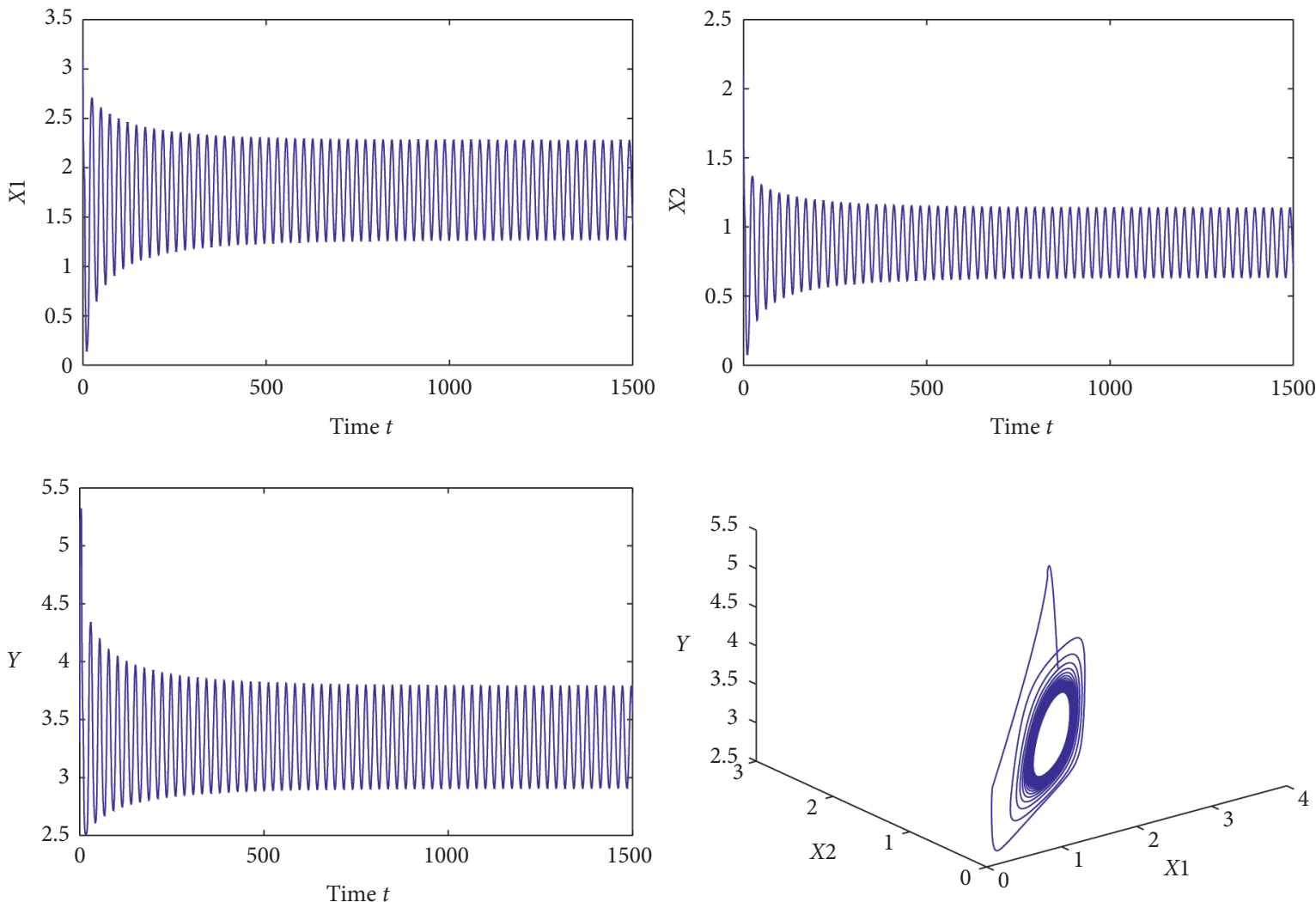

Figure 3: $\tau=4.25>\tau_{0}$. The bifurcation periodic solution is stable. 
Theorem 3. From (75), one has

(1) The directions of the Hopf bifurcation is determined bysign $\left(\mu_{2}\right)$ : if $\mu_{2}>0\left(\mu_{2}<0\right)$, then the Hopf bifurcation is forward (backward) and the bifurcating periodic solutions exist for $\tau>\tau_{0}\left(\tau<\tau_{0}\right)$.

(2) The stability of the bifurcating periodic solutions is determined bysign $\left(\beta_{2}\right)$ : the bifurcating periodic solutions are stable (unstable) if $\beta_{2}<0\left(\beta_{2}>0\right)$.

(3) The period of the bifurcating periodic solutions is determined bysign $\left(T_{2}\right)$ : the period increases (decreases) if $T_{2}>0\left(T_{2}<0\right)$.

\section{Numerical Examples}

In this section, a numerical example is presented to support our obtained results. Consider system (3) with the following parameters: $r_{1}=r_{2}=r_{3}=1, c_{1}=0.05, c_{2}=0.081, d_{1}=$ $d_{2}=d=0.01, a_{1}=0.2, a_{2}=0.3, a_{3}=0.4, b_{1}=0.1$, and $b_{2}=0.1$. The positive equilibrium of (3) is $(1.8155$, $0.9077,3.3149)$. By (17), we can obtain $\tau_{0}=4.15$.

First, we choose $\tau=4.1<\tau_{0}$, and the outputs of three enterprises are shown in Figure 2. It is easy to see that output of enterprise is asymptotically stable.

Finally, we choose $\tau=4.25>\tau_{0}$, and the outputs of three enterprises are shown in Figure 3; it is easy to see that the output of the enterprise displays a periodic fluctuation.

\section{Economic Meaning}

In this paper, a novel competition-cooperation enterprise cluster model with core-satellite structure is proposed. The boundedness of the positive equilibrium is investigated. It is found that there exists upper bound of both core enterprise output and satellite enterprise output and the upper bound of core enterprise not only depends on its own production capacity but also depends on the production capacity of two satellite enterprises. Moreover, it is found that the production period plays a key role on the evolution of enterprise cluster. From simulation, we can obtain that the critical value of the production period is $\tau_{0}=4.15$. When the production period passes $\tau_{0}=4.15$, the output of the enterprise cluster system loses its stability and displays a periodic fluctuation, which means too long production cycle will lead to capacity fluctuations. From the viewpoint of enterprise management, output fluctuation may affect the stable development of enterprises, worker employment, and production efficiency. Thus, it is important for enterprise cluster to control the production period in a suitable region.

\section{Data Availability}

The data used to support the findings of this study are available from the corresponding author upon request.

\section{Conflicts of Interest}

The authors declare that they have no conflicts of interest.

\section{Acknowledgments}

This study was supported in part by the Chongqing Social Science Planning Project under grant no. 2019BS053, in part by the Science and the Technology Research Program of Chongqing Municipal Education Commission under grant no. KJQN201900816, in part by the Social Science Research Program of Chongqing Municipal Education Commission under grant no. 20SKJD072 in part by the Research Funds of Chongqing Technology and Business University under grant no. 1955028, and in part by the Chongqing Basic and Frontier Research Project under grant nos. cstc2019jcyjmsxmX0470 and cstc2020jcyj-msxmX0139.

\section{References}

[1] W. Souza, G. Scur, and W. Hilsdorf, "Eco-innovation practices in the brazilian ceramic tile industry: the case of the Santa Gertrudes and Criciúma clusters," Journal of Cleaner Production, vol. 199, pp. 1007-1019, 2018.

[2] G. Buenstorf and C. Costa, "Drivers of spin-off performance in industry clusters: embodied knowledge or embedded firms?" Research Policy, vol. 47, no. 3, pp. 663-673, 2018.

[3] S. W. Ximenes, S. Roberts, T. S. Lee, H.-S. Shin, B. Foing, and C. Duarte, "LEAP2 and LCATS industry clusters: a framework for lunar site technology development using global, spaceSTEM education and global space-industry development networks," Acta Astronautica, vol. 157, pp. 61-72, 2019.

[4] N. L. Duc and J. Lindeque, "Proximity and multinational enterprise co-location in clusters: a multiple case study of Dutch science," Industry \& Innovation, vol. 25, no. 3, pp. 282-307, 2018.

[5] R. Zhou, "Empirical Analysis of the Impact Which the Shared Resources Have on the Competitive Advantages of the Logistics Enterprise in Logistics Industry Clusters," Geo-Informatics in Resource Management and Sustainable Ecosystem, pp. 60-69, 2017.

[6] D. Janasová, V. Bobáňová, and S. Strelcová, "Networking of small and medium enterprises into clusters in the Slovak republic," Procedia Engineering, vol. 192, pp. 370-375, 2017.

[7] L. Chen, Y. Zhou, D. Zhou, and L. Xue, "Clustering enterprises into eco-industrial parks: can interfirm alliances help small and medium-sized enterprises?" Journal of Cleaner Production, vol. 168, pp. 1070-1079, 2017.

[8] J. Zhao, C. Luo, and Y. Zheng, "Evolutionary dynamics of the cooperation clusters on interdependent networks," Physica A: Statistical Mechanics and Its Applications, vol. 517, pp. 132140, 2019.

[9] L. Trujillo, J. Campos, and I. Pérez, "Competition vs. cooperation between neighbouring ports: a case study in Chile," Research in Transportation Business \& Management, vol. 26, pp. 100-108, 2018.

[10] C. $\mathrm{Xu}$, "Periodic behavior of competition and corporation dynamical model of two enterprises on time scales," Journal of Quantitative Economics, vol. 29, no. 2, pp. 1-4, 2012.

[11] D. Jana, P. Dolai, A. K. Pal, and G. P. Samanta, "On the stability and Hopf-bifurcation of a multi-delayed competitive population system affected by toxic substances with imprecise biological parameters," Modeling Earth Systems and Environment, vol. 2, pp. 110-127, 2016.

[12] G. P. Samanta, "A two-species competitive system under the influence of toxic substances," Applied Mathematics and Computation, vol. 216, no. 1, pp. 291-299, 2010. 
[13] A. K. Pal, P. Dolai, and G. P. Samanta, "Dynamics of a delayed competitive system affected by toxic substances with imprecise biological parameters," FILOMAT, vol. 31, no. 16, pp. 5271-5293, 2017.

[14] X. Yang, Z. Zhu, H. Yu, Y. Zhao, and L. Guo, "Evolutionary Game Dynamics of the Competitive Information Propagation on Social Networks," Complexity, vol. 2019, Article ID 8385426, 11 pages, 2019.

[15] Z. Liu, Y. Liu, and H. Li, "A Coordination Mechanism of Supply Chain with a Retailer and Two Competitive Suppliers," Complexity, vol. 2020, Article ID 2128497, 14 pages, 2020.

[16] H. Zhou, "Enterprises cluster co-existence model and stability analysis," Systems Engineering, vol. 21, no. 4, pp. 32-37, 2003.

[17] Q. Guo, "Competitive strategies in an enterprise: an ecological model," Geological Technoeconomic Management, vol. 27, no. 2, pp. 49-52, 2005.

[18] Z. Wang and R. Pan, "Analysis to the equilibrium of industrial clusters based on ecological model," Statistical Thinktank, vol. 8, pp. 32-35, 2008.

[19] M. Liao, C. Xu, and X. Tang, "Dynamical behaviors for a competition and cooperation model of enterprises with two delays," Nonlinear Dynamics, vol. 75, no. 1-2, pp. 257-266, 2014.

[20] M. Liao, C. Xu, and X. Tang, "Stability and Hopf bifurcation for a competition and cooperation model of two enterprises with delay," Communications in Nonlinear Science and $\mathrm{Nu}$ merical Simulation, vol. 19, no. 10, pp. 3845-3856, 2014.

[21] Y. Li and T. Zhang, "Global asymptotical stability of a unique almost periodic solution for enterprise clusters based on ecology theory with time-varying delays and feedback controls," Communications in Nonlinear Science and Numerical Simulation, vol. 17, no. 2, pp. 904-913, 2012.

[22] W. Hu, H. Zhao, and T. Dong, "Dynamic analysis for a kaldor-kalecki model of business cycle with time delay and diffusion effect," Complexity, vol. 2018, Article ID 1263602, 11 pages, 2018.

[23] T. Dong and Q. Zhang, "Stability and oscillation analysis of a gene regulatory network with multiple time delays and diffusion rate," IEEE Transactions on NanoBioscience, vol. 19, no. 2, pp. 285-298, 2020.

[24] T. Dong and L. Xia, "Spatial temporal dynamic of a coupled reaction-diffusion neural network with time delay," Cognitive Computation, vol. 11, no. 2, pp. 212-226, 2019.

[25] S. Ruan and J. Wei, "On the zeros of transcendental functions with applications to stability of delay differential equations with two delays," Dynamics.of Continous Discrete Impulsive Systems Series. Applications and Algorithms.vol. 10, pp. 863873, 2003.

[26] B. Hassard, N. Kazarinoff, and Y. Wan, Theory and Applications of Hopf Bifurcation, Cambridge University Press, Cambridge, UK, 1981.

[27] X. Zhang and Z. Liu, "Hopf bifurcation analysis in a predatorprey model with predator-age structure and predator-prey reaction time delay," Applied Mathematical Modelling, vol. 91, pp. 530-548, 2021.

[28] X. Zhang and Z. Liu, "Periodic oscillations in HIV transmission model with intracellular time delay and infection-age structure," Communications in Nonlinear Science and Numerical Simulation, vol. 91, Article ID 105463, 2020. 